SHORT REPORT

\title{
DNA end labelling (TUNEL) in a 3 year old girl with Leigh syndrome and prevalent cortical involvement
}

\author{
P Formichi, A Malandrini, C Battisti, F M Santorelli, S Gambelli, S A Tripodi, G Berti, C Salvadori, \\ A Tessa, A Federico
}

J Neurol Neurosurg Psychiatry 2004;75:930-932. doi: 10.1136/jnnp.2003.016410

\begin{abstract}
Neuropathological study of a $3 \frac{1}{2}$ year old girl with familial Leigh syndrome who also harboured a rare ATPase gene mutation disclosed extensive and unusual lesions in the cerebral cortex, despite a typical histological pattern. Early lesions in the periacqueductal grey matter of the brainstem, characterised by capillary congestion and initial regressive neuronal changes, were also observed, along with TUNEL reactive neuronal cells showing morphological signs typical of apoptosis in cortical areas with neuronal cell loss. The finding of lesions in atypical brain areas and for the first time, very early regressive neuronal phenomena, suggest that early changes in crucial brain areas may have been a cause of death. The abundance of TUNEL positive nuclei in cortical areas in the present case suggests that the apoptosis may be involved in the mechanism of neuronal death in Leigh syndrome.
\end{abstract}

p eigh syndrome (LS) is a sub-acute necrotising encephalopathy of infancy or early childhood. Recessive and X linked inheritance patterns have been described in association with mutations in genes encoding structural components of mitochondrial oxidative phosphorylation pathways or proteins necessary for their correct assembly. ${ }^{1}$ Mutations of mtDNA are a common cause of LS. ${ }^{1}$ Despite its multiform pathogenesis, LS has stereotypical neuropathological findings consisting of symmetrical necrotic lesions in the brainstem, diencephalon, cerebellum, and spinal cord. ${ }^{2}$ Here we report unusual pathological features in an LS child harbouring a rare mtDNA mutation in the ATPase 6 subunit gene.

\section{METHODS}

Detailed clinical and molecular genetic findings have been reported elsewhere. ${ }^{3}$ Briefly, the proband was born at term by caesarean section. She presented severe developmental delay. At 3 years of age, neurological examination showed oligophrenia, strabismus, and ataxic-spastic gait. She was only able to pronounce simple words. Relatives reported severe epilepsy with 3-4 epileptic fits per week. During hospitalisation, repeated assay of serum lactic acid revealed a persistent lactic acidosis. The child died of cardiorespiratory arrest at $3 \frac{1}{2}$ years of age. Her 10 year old sister is affected with the same disease. Molecular genetic analysis disclosed an mtDNA mutation (T9176G) in the ATPase 6 gene. ${ }^{3}$

\section{Neuropathological study}

Autopsy was perfomed 10 hours after death, and the brain fixed in formalin for 30 days. Samples from representative areas were embedded in paraffin and stained by the following techniques: haematoxylin and eosin, Nissl, Luxol fast blue, Bodian, and PAS. We performed an immunocytochemical analysis using the peroxidase-antiperoxidase method. Colour was developed using 3, 3'-diaminobenzidine (Sigma) as chromogen. Sections were observed under a Zeiss Axiomat Optical microscope. Paraffin sections were incubated with antibodies for MBP (Biogenex, USA), chromogranin A, synaptophysin, NSE, and GFAP (all Dakopatts, Denmark). Brain tissue from two young girls aged 6 and 9 years, who had died of extraneurological causes, was used as control.

\section{Apoptosis study}

Apoptosis and DNA fragmentation were detected in tissue sections by terminal deoxynucleotidyl transferase mediated dUTP nick end labelling (TUNEL) assay and Hoechst staining. TUNEL was also used to detect nuclear DNA fragmentation in situ. Paraffin embedded sections from cortical areas were deparaffinised, and 3'-OH terminal DNA fragments were then labelled with an In Situ Cell Death Detection POD kit (Boehringer Mannheim, Italy). For each specimen, positive (incubating a brain section for 10 minutes with DNAse I before TUNEL reaction mixture) and negative (incubating a muscle section without TdT) controls were performed.

Hoechst $\mathrm{H} 33258$ binds to DNA and reveals nuclear condensation and fragmentation associated with apoptosis. ${ }^{4}$ Deparaffinised sections of cortical areas were stained with Hoechst $\mathrm{H} 33258$ ( $1 \mu \mathrm{g} / \mathrm{ml}$ in PBS) for 30 minutes at room temperature and examined by fluorescence microscopy. Brain tissue from patient who had died of a heart attack was used as control.

\section{RESULTS}

\section{Neuropathological study}

In the right hemisphere, the cortex of the posterior frontal convolutions had a necrotic appearance with dark reddish brown discoloration. In the left hemisphere, a zone of cortical necrosis was observed in the occipital lobe. Histologically, the affected cortex was clearly delineated from the subcortical white matter by a wall of reactive astrocytes (gemistocytes). The cortical lesions consisted of spongy vacuolation with areas of cystic cavitation; there were many macrophages, reactive astrocytes, and hypertrophic congested blood vessels (fig IC,D). There was severe neuronal loss and many neurones in affected areas stained darkly, giving the appearance of incrustations (fig 1A,B). The leptomeningeal vessels covering affected convolutions were thickened and congested (fig lA). In the pons and midbrain, around the periaqueductal grey matter, we observed symmetrical areas of capillary congestion and initial spongy vacuolation; in these areas, neurones showed chromatolytic changes characterised by a rounded cell body, disappearance of dendritic processes,

Abbreviations: LS, Leigh syndrome; MILON, mitochondrial late onset neurodegeneration; TUNEL, terminal deoxynucleotidyl transferase mediated dUTP nick end labelling 
and unrecognisable Nissl bodies (fig IC). We observed similar findings in the right putamen. In the cerebellum, there was slight loss of Purkinje cells with some torpedo formations and proliferation of Bergman glia (fig 1D). The cerebellar white matter showed moderate astrocytosis. No abnormality was found in the dentate, inferior olivary nuclei, and hippocampus.

By GFAP immunocytochemistry, we observed a severe astrocytosis surrounding the cortical lesions (fig 1E). GFAP positive cells were also observed in the white matter of cerebellum, mostly subcortical, and in the brainstem. The immunocytochemical study by MBP revealed reduced staining in the areas of white matter surrounding the cortical lesions and in the cerebellum. The reaction with chromogranin A and synaptophysin gave weak staining in grey matter regions without significant difference with respect to controls and was almost absent in the affected cortex.

All representative areas (frontal and occipital cortex, cerebellum, basal ganglia, pons, and midbrain) harboured high levels $(>95 \%)$ of mutant mtDNA.

\section{Apoptotic study}

The frontal and occipital cortical brain regions with neural loss tested for TUNEL reaction showed a greater number of TUNEL positive neurones than controls (fig 2A). The same results were obtained using Hoechst H33258 staining as a complementary method to detect apoptotic death. The control brain showed negative H33258 labelling, whereas the LS brain showed an abundance of H33258 positive fragmented nuclei (data not shown). TUNEL reaction performed in sections tissue of the cerebellum, basal ganglia, and inferior olivary nuclei showed TUNEL negative labelling (fig $2 \mathrm{~B}-\mathrm{D}$ ).

\section{DISCUSSION}

It is still unclear how and why neurones die in mitochondrial disorders. The ATP compartmentation theory proposed for pathogenic mtDNA mutation ${ }^{5}$ suggests that cytosolic ATP derived from glycolysis cannot substitute for mitochondrion derived oxidative ATP in affected brain areas to perform the important tasks of running channels and pumping neurotransmitters. This probably occurs because there is too little ATP or the ATP is unavailable. Because in maternally inherited diseases resulting from pathogenic mutations in mtDNA, normal functioning of the respiratory chain and OXPHOS is impaired, a causal relationship between mitochondrial dysfunction and neurodegeneration via apoptosis would be expected. Although apoptosis detected by TUNEL has been described in human brains affected by neurodegenerative diseases, ${ }^{467}$ no data are available on patients with Leigh syndrome or other mitochondrial encephaloneuromyopathies, and the relationship between apoptosis and mitochondrial diseases is still contradictory. Experimentally, in early and end stage symptomatic (age 5-6 months) mitochondrial late onset neurodegeneration (MILON) mice, enhanced presence of TUNEL reactive neuronal cells showing typical morphological features of apoptosis was recently reported in all brain areas with cell loss. ${ }^{8}$ The same authors showed an absence of TUNEL positive nuclei in 2, 3, and 4 month old presymptomatic MILON mice and an increase in apoptotic nuclei after kanainic acid injection ${ }^{8}$ suggesting that prolonged neurone chain deficiency is required for induction of neurodegeneration and that respiratory chain deficient neurones are more vulnerable to excitotoxic challenge. Our results, obtained for the first time in a brain of an LS patient, are in line with this recent experimental data, ${ }^{8}$ showing a large number of TUNEL positive nuclei in frontal and

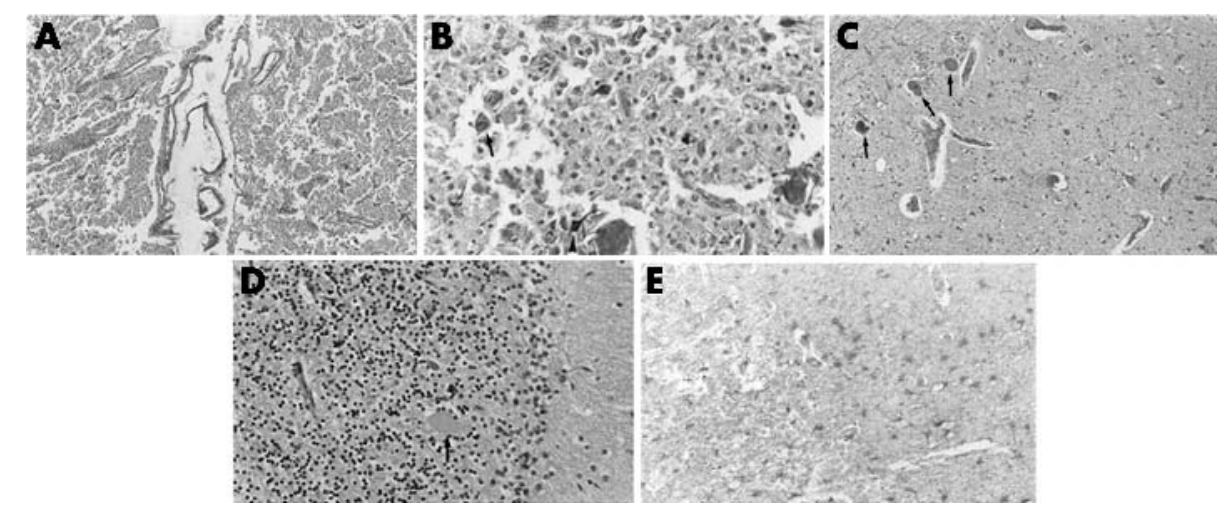

Figure 1 (A) Two adjoining frontal cortical convolutions showing spongy necrosis with areas of cavitation, many macrophages, and vascular proliferation. Note some neurones with incrustation and thickened congested meningeal vessels. (B) Histological preparation of cortical lesions in frontal convolutions showing spongy cavitated necrosis with many macrophages and reactive astrocytes. Note surviving neurones (arrow) and degenerating neurones with incrustation (arrowhead). (C) Initial vascular congestion, slight spongiosis, and chromatolytic neurones (arrows) in the periacqueductal region. (D) Cerebellar cortex showing Purkinje cell loss and Bergman glia proliferation. Note large torpedo formation (arrow). (E) Many GFAP positive cells in areas surrounding a cortical lesion in the brain cortex. Haematoxylin and eosin staining, original magnification: $A, \times 80 ; B, \times 400 ; C, \times 120 ; D, \times 350 ; E, \times 300$.
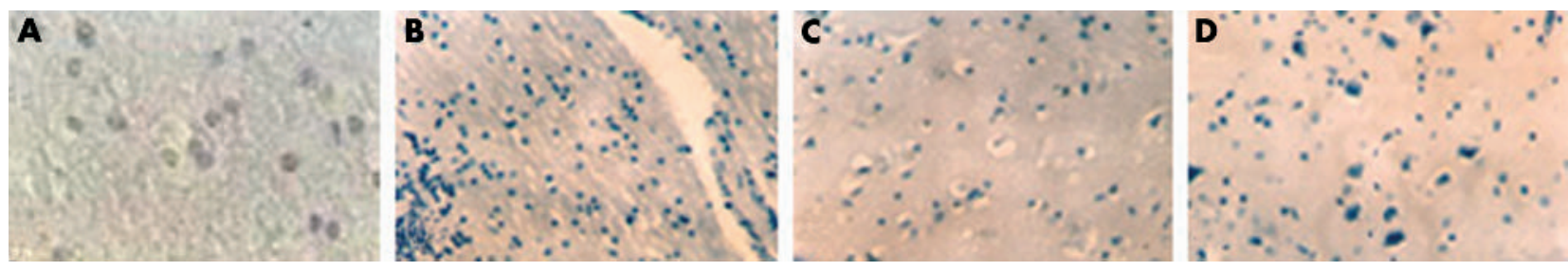

Figure 2 Sections from LS brain analysed by TUNEL assay. (A) Several TUNEL positive neurones were detected in the cortex area; (B-D) TUNEL reaction performed in sections tissue of the cerebellum (B), basal ganglia (C), and inferior olivary nuclei (D) showed TUNEL negative labelling. Original magnification $\times 400$. 
occipital cortical area of the patient. This finding showed that the apoptotic process is responsible for neuronal loss in LS patients and confirms that in neurodegenerative diseases, such as LS, a mutation in mtDNA could be linked to apoptosis in specific populations of neurones.

In the present case, we did not find the pathological features of LS in typical sites such as the brainstem, cerebellum, thalamus, and basal ganglia. Furthermore, we observed some peculiar aspects, difficult to interpret. (a) To our knowledge, the cerebral cortex has never been reported to be affected to this extent in $\mathrm{LS}^{;}{ }^{2}{ }^{9}$ our patient showed widespread severe cortical neuronal loss. In a cohort of 21 autopsies, Cavanagh \& Hardin found mild cortical neuronal loss only in one case. Necrosis of the cerebral cortex in mitochondrial diseases may be due to episodes of hypotension before death or postconvulsive damage. The present patient suffered from epileptic fits but it is difficult to determine whether they were responsible for cortical damage. With respect to classical cortical LS lesions, there were also cystic cavities and a regressive appearance of the neurones, referred to as incrustations, indicating that this type of lesion occurred some time before death. (b) Brain lesions are always symmetrical in LS, but in our case they involved the right and left hemisphere asymmetrically. (c) In reported LS cases with mutation at nt9176 of the ATPase gene, lesions have always been found in typical regions of the brain. ${ }^{10-12}$

The lesions we observed in the periacqueductal regions and putamen are rarely found in LS, but they are important for understanding the sequence of pathological events leading to classical abiotrophic lesions. Vascular congestion, which may be due to increased lactate concentrations in neutrophils, seems to be the primary event. In two different studies, Cavanagh $\mathrm{et} \mathrm{al}^{2}{ }^{13}$ hypothesised that the main involvement of the brainstem, cerebellar white matter, and basal ganglia in LS is related to their greater vulnerability to increased levels of lactic acid, irrespective of the genetic defect, and probably secondary to the type of supportive vascular supply, provided in these regions by perforant arterioles. The finding of damaged neurones in these lesions suggest that neuronal changes may be an early phenomenon in LS. This is in line with localised proton magnetic resonance spectroscopy evidence of early neuronal loss and breakdown of membrane phospholipids observed in a LS/T8993G patient. ${ }^{14}$

In conclusion, our study confirms that metabolic damage to nervous tissue observed in autopsy specimens of LS patient may not be associated with appreciable morphological changes for a long time. Indeed, the symmetrical brainstem and basal ganglia lesions, considered to be pathognomonic of this syndrome, usually occur in the last stages and are responsible for respiratory distress and death. In the present case, the very early changes in the midbrain were probably the cause of death.

\section{ACKNOWLEDGEMENTS}

This research was partly financed by a grant from the Ministry of Health (Rome) to A Federico.

\section{Authors' affiliations}

P Formichi, A Malandrini, C Battisti, S Gambelli, G Berti, C Salvadori,

A Federico, Department of Neurological and Behavioural Sciences, Section of Neurological Sciences, University of Siena, Siena, Italy

P Formichi, Anni Verdi Association, Rome, Italy

S A Tripodi, Department of Pathology, University of Siena, Siena, Italy

F M Santorelli, A Tessa, Molecular Medicine, IRCCS-Bambino Gesù

Children's Hospital, Rome, Italy

Competing interests: none declared

Correspondence to: Dr A Malandrini, Department of Neurological and Behavioural Sciences, Viale Bracci 2, 53100 Siena, Italy; malandrini@ unisi.it

Received 8 April 2003

In revised form 6 October 2003

Accepted 12 October 2003

\section{REFERENCES}

1 DiMauro S, De Vivo DC. Genetic heterogeneity in Leigh Syndrome. Ann Neurol 1996;40:5-7

2 Cavanagh JB, Harding BN. Pathogenic factors underlying the lesions in Leigh's disease. Brain 1994; 117:1357-76.

3 Carrozzo R, Tessa A, Vaquez-Memije ME, et al. The T9176G mtDNA mutation severely affects ATP production and results in Leigh syndrome. Neurology 2001;56:687-90.

4 de la Monte SM, Luong Y, Neely TR, et al. Mitocondrial DNA damage as a mechanism of cell loss in Alzheimer's disease. Lab Invest 2000;80:1323-36.

5 Schon EA. Mitochondrial genetics and disease. Trends Biochem Sci 2000;25:555-60.

6 de la Monte SM, Sohn YK, Ganju N, et al. p53- and CD95-associated apoptosis in neurodegenerative diseases. Lab Invest 1998;78:401-11.

7 Thomas LB, Gates DJ, Richfield E, et al. DNA end labeling (TUNEL) in Huntington's disease and other neuropathological conditions. Exp Neurol 1995; 133:265-72.

8 Sörensen L, Ekstrand M, Silva JP, et al. Late-onset corticohippocampal neurodepletion attributable to catastrophic failure of oxidative phosphorylation in MILON mice. J Neurosci 2002;21:8082-98.

9 Montpetit VJ, Andermann F, Carpenter S, et al. Subacute necrotizing encephalomyelopathy: a review and a study of two families. Brain 1971;94:1-30.

10 Campos Y, Martin MA, Rubio JC, et al. Leigh syndrome associated with the T9176C mutation in the ATPase 6 gene of mitochondrial DNA. Neurology 1997;49:595-7.

11 Dionisi-Vici C, Seneca S, Zeviani M, et al. Fulminant Leigh syndrome and sudden unexpected death in a family with the T9176C mutation of the mitochondrial ATPase 6 gene. J Inherit Metab Dis 1998;21:2-8.

12 Michiko M, Satoshi H, Yu-ichi G, et al. Confirmation that a T-to-C mutation at 9176 in mitochondrial DNA is an additional candidate mutation for Leigh's syndrome. Neuromusc Disord 1998;8:149-51.

13 Cavanagh JB. Selective vulnerability in acute energy deprivation syndromes. Tissue responses to cellular energy deprivation and their clinico-pathological consequences. Neuropathol Appl Neurobiol 1993;19:461-70.

14 Takahashi S, Oki J, Miyamoto A, et al. Proton magnetic resonance spectroscopy to study the metabolic changes in the brain of a patient with Leigh syndrome. Brain Dev 1999;21:200-4. 